\title{
Three-dimensional assessment of bronchiectasis in a mouse model of mucociliary clearance disorder
}

To the Editor:

Bronchiectasis is a chronic pathological condition characterised by abnormal enlargement of the lung's conductive airways. It is associated with a lack of ciliary motility and restricted mucociliary clearance in diseases such as primary ciliary dyskinesia (PCD) or "immotile cilia syndrome". Recent studies have shown an increase in the prevalence of bronchiectasis, causing a significant burden on public healthcare systems $[1,2]$. The mechanisms that trigger and drive the development of bronchiectasis have yet to be fully elucidated. Murine models of immotile cilia or reduced mucociliary clearance failed to display signs of bronchiectasis in multiple studies, raising questions about the suitability of murine models for non-cystic fibrosis (CF) bronchiectasis and hindering the development of targeted therapies [3].

The influence of age and size has been discussed since the duration of mucociliary clearance defects could significantly impact airway remodelling, and many murine models of PCD do not survive long enough for the development of relevant airway remodelling [4]. Moreover, the murine airway anatomy poses significant challenges to the employed imaging modality since most of the conducting airways measure under $1 \mathrm{~mm}$ in diameter [5]. Motion artefacts from ventilation and the beating heart further limit the imaging results; they were named the number one technical pitfall in the computed tomography (CT) evaluation of bronchiectasis [6]. Given these limitations, the failure in developing suitable murine models of immotile cilia raises the question: "Could we have simply missed it?"

To answer the question of whether we can detect a phenotype of bronchiectasis or bronchial enlargement under pathogen-free conditions in laboratory mice with mucociliary clearance defects, we generated a cohort of TAp73 wildtype (WT, $n=6)$ and knockout $(\mathrm{KO}, \mathrm{n}=4)$ mice. All experimental procedures were approved and performed in accordance with the requirements set forth by the Ethics Committee of the University Medical Centre Goettingen (application number: 18/2/16).

TP73, a p53 homologue, has been identified in key steps during the differentiation of motile multiciliated cells and the regulation of airway multiciliogenesis, and TAp73 KO mice have strongly reduced mucociliary clearance [7-9]. Mice were evaluated at 10 months of age (308 days), corresponding to a human age of $\sim 34$ years [10]. At this age, most human patients with severe PCD have already developed visible bronchiectasis on chest CT. Synchrotron-based imaging has been shown to increase structural detectability and permit exact quantification of tissue parameters in small rodents in a variety of applications and organ systems. The high degree of coherence of synchrotron X-ray light allows for phase-sensitive imaging strategies that cannot be applied using commonly available X-ray tubes [11]. One of those is the so-called synchrotron free-propagation phase-contrast CT (SR-pCT), which is characterised by a larger contrast-to-noise ratio in soft-tissue application than classical CT and has been successfully applied for lung imaging in a multitude of studies $[12,13]$. The ability of SR-pCT to study the mouse lung in situ in great detail enables reliable depiction of subtle anatomical alterations in their original context [13].

@ERSpublications

Synchrotron-based imaging allows for detection of bronchiectasis-like phenotypes in mice with mucociliary clearance disorders https://bit.ly/3gXGdP3

Cite this article as: Wagner W, Dullin C, Andreas S. Three-dimensional assessment of bronchiectasis in a mouse model of mucociliary clearance disorder. ERJ Open Res 2021; 7: 006352020 [https://doi.org/10.1183/23120541.00635-2020].

Copyright @ERS 2021. This article is open access and distributed under the terms of the Creative Commons Attribution NonCommercial Licence 4.0. 


\section{Lessons for clinicians}

Murine models of reduced mucociliary clearance have been established to investigate their pathobiology and develop novel treatment approaches but failed to develop visible airway enlargements. Synchrotron free-propagation phase-contrast computed imaging is an innovative, sensitive, nondestructive in situ technique that allows for three-dimensional ultra-high-resolution detection of bronchiectasis in murine models of impaired mucociliary clearance.

Here we utilised SR-pCT to analyse the lung structure at a spatial resolution of $\sim 9 \mu \mathrm{m}$. To maintain the lung architecture, sacrificed mice were tracheotomised and the lungs were inflated with a constant air pressure of $30 \mathrm{cmH}_{2} \mathrm{O}$. The trachea was tied, and the entire mouse was embedded in $1 \%$ agarose gel in 50-mL falcon tubes to prevent post mortem deformation or lung deflation [14]. Imaging was performed at the SYRMEP beamline of the Italian synchrotron light source "Elettra" Trieste, Italy using a sample-to-detector distance of $30 \mathrm{~cm}$, a quasi-monochromatic $\mathrm{x}$-ray beam with an energy of $22 \mathrm{keV}$ and a full $360^{\circ}$ off-centre scan was performed yielding 1800 angular projections by an effective detector pixel size of $9 \times 9 \mu \mathrm{m}^{2}$. Single-distance phase retrieval was applied using the transport of intensity equation [15]. 3D reconstructions were performed utilising a classical filtered back projection algorithm. The delta-to-beta ratio between the decrement of the real part of the complex refractive index and the imaginary part was set to 1950 based on Monammadi et al. [16]. In the obtained 3D data sets 5-10 volumes-of-interest of a size of $22 \mathrm{~mm}^{3}$ were randomly selected in the periphery of the lung. The average pore size of small conducting airways was then quantified in three dimensions utilising the software "Pore3D", resulting in a list of pore descriptions for each volume-of-interest. To focus the analysis to smaller bronchi rather than larger airways or parenchymal alveoli, only pores with a size between 0.2 and $0.75 \mathrm{~mm}$ were used to assess bronchial dilation. To minimise the influence of differences in the size and weight of the analysed mice we normalised the values with the 3rd root of the weight.

Using SR-pCT we were able to analyse the entire mouse lung in situ on the anatomical level of small conducting airways and found significantly larger normalised airway diameters in the TAp73 KO compared to that found in the WT mice. Side-by-side comparison of a WT control (figure 1a) and a TAp73 KO mouse (figure $1 \mathrm{~b}$ ) shows enlarged small airways in the $\mathrm{KO}$, suggesting the appearance of bronchiectasis-like phenotype in those mice. Statistical evaluation of the cohorts revealed higher porosity of the TAp73 KO lungs with reduced mucociliary clearance.

a)

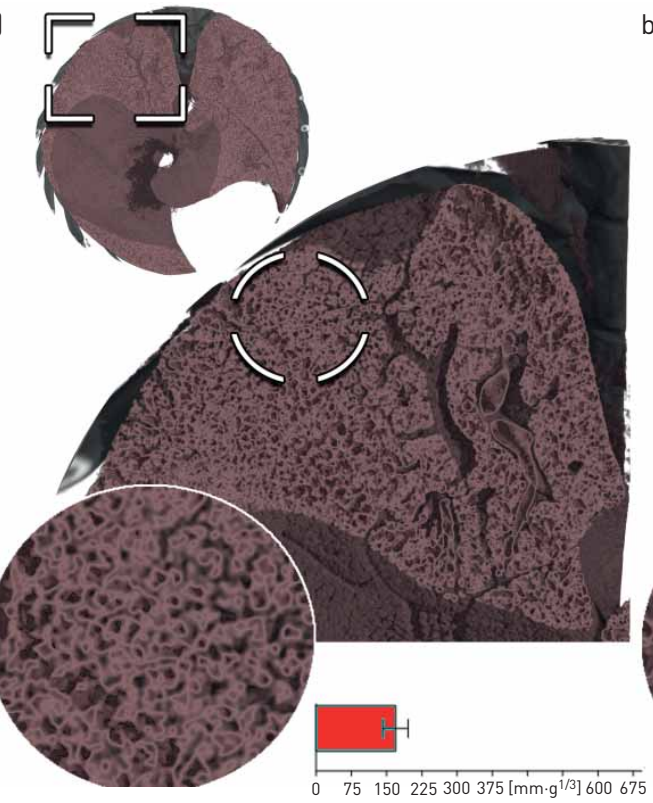

$2.5 \times$

Normalised avg. pore size

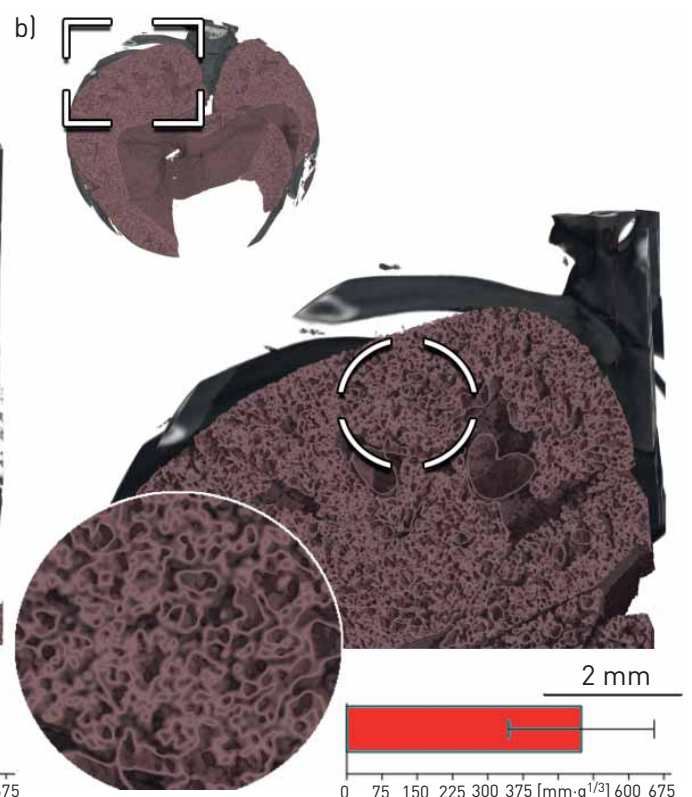

Normalised avg. pore size

FIGURE 1 Synchrotron free-propagation phase-contrast computed tomography in situ analysis (spatial resolution of $\sim 9 \mu \mathrm{m}$ ) of al a wildtype (WT) control and b) a TAp73 knockout (KO) mouse with mucociliary clearance defects. Cross-sections of the entire lung are displayed with two levels of magnification of the left lower lobe $(p<0.05)$. The depicted bars present the results for TAp73 KO $(n=4) 499 \pm 310 \mathrm{~mm} \cdot \mathrm{g}^{-1 / 3}$ and WT $(\mathrm{n}=6)$ $169 \pm 66 \mathrm{~mm} \cdot \mathrm{g}^{-1 / 3}(\mathrm{mean} \pm \mathrm{sD})(\mathrm{p}<0.032)$. 
Our study demonstrates that SR-pCT is a sensitive, nondestructive in situ technique for three-dimensional ultra-high-resolution detection of bronchiectasis in mouse models of immotile cilia and restricted mucociliary clearance. Additionally, we show that mice with defective mucociliary clearance do develop measurable airway enlargements at 10 months, corresponding to the mid-thirties in humans. Therefore, SR-pCT may be a useful tool for pre-clinical and translational evaluation of novel treatment strategies and may allow for small rodent research to broaden our understanding of the mechanisms involved in onset and development of airway remodelling and bronchiectasis.

\section{Willi Wagner ${ }^{1,2}$, Christian Dullin ${ }^{2,3}$, Stefan Andreas $\oplus^{4}$ and Muriel Lizé $\oplus^{4}$}

${ }^{1}$ University of Heidelberg, Dept of Diagnostic and Interventional Radiology, Translational Lung Research Center Heidelberg (TLRC), German Center for Lung Research (DZL), Heidelberg, Germany. ${ }^{2}$ University Medical Center Goettingen, Institute for Diagnostic and Interventional Radiology, Goettingen, Germany. ${ }^{3}$ Max-Planck-Institute for Experimental Medicine, Goettingen, Germany. ${ }^{4}$ Bayer AG, Cardiovascular Research, Lung Diseases, Wuppertal, Germany.

Correspondence: Muriel Lizé, University Medical Center Goettingen, Clinic for Cardiology and Pneumology, Molecular \& Experimental Pneumology, Justus-von-Liebig-Weg 11, GZMB Goettingen 37077, Germany. E-mail: mlize@gwdg.de

Received: 1 Sept 2020 | Accepted after revision: 7 Dec 2020

Conflict of interest: W. Wagner has nothing to disclose. C. Dullin has nothing to disclose. S. Andreas reports personal fees from Boehringer Ingelheim and GlaxoSmithKline, and payments for presentations from AstraZeneca, Berlin Chemie, Chiesi and Novartis, outside the submitted work. M. Lizé is an employee of Bayer AG.

\section{References}

1 Diel R, Chalmers JD, Rabe KF, et al. Economic burden of bronchiectasis in Germany. Eur Respir J 2019; 53: 1802033.

2 Ringshausen FC. Increasing bronchiectasis prevalence in Germany, 2009-2017: a population-based cohort study. Eur Respir J 2019; 54: 1900499.

3 Norris DP, Grimes DT. Mouse models of ciliopathies: the state of the art. Dis Model Mech 2012; 5: 299-312.

4 Song R, Walentek P, Sponer $\mathrm{N}$, et al. miR-34/449 miRNAs are required for motile ciliogenesis by repressing cp110. Nature 2014; 510: 115-120.

5 Thiesse J, Namati E, Sieren JC, et al. Lung structure phenotype variation in inbred mouse strains revealed through in vivo micro-CT imaging. J Appl Physiol (1985) 2010; 109: 1960-1968.

6 McGuinness G, Naidich DP, Leitman BS, et al. Bronchiectasis: CT evaluation. AJR Am J Roentgenol 1993; 160: 253-259.

7 Marshall CB, Mays DJ, Beeler JS, et al. p73 is required for multiciliogenesis and regulates the Foxj1-associated gene network. Cell Rep 2016; 14: 2289-2300.

8 Nemajerova A, Kramer D, Siller SS, et al. TAp73 is a central transcriptional regulator of airway multiciliogenesis. Genes Dev 2016; 30: 1300-1312.

9 Wildung M, Esser TU, Grausam KB, et al. Transcription factor TAp73 and microRNA-449 complement each other to support multiciliogenesis. Cell Death Differ 2019; 26: 2740-2757.

10 Dutta S, Sengupta P. Men and mice: relating their ages. Life Sci 2016; 152: 244-248.

11 Momose A, Takeda T, Itai Y, et al. Phase-contrast X-ray computed tomography for observing biological soft tissues. Nat Med 1996; 2: 473-475.

12 Kitchen MJ, Buckley GA, Gureyev TE, et al. CT dose reduction factors in the thousands using X-ray phase contrast. Sci Rep 2017; 7: 1-9.

13 Dullin C, Dal Monego S, Larsson E, et al. Functionalized synchrotron in-line phase-contrast computed tomography: a novel approach for simultaneous quantification of structural alterations and localization of barium-labelled alveolar macrophages within mouse lung samples. J Synchrotron Rad 2015; 22: 143-155.

14 Dullin C, Larsson E, Tromba G, et al. Phase-contrast computed tomography for quantification of structural changes in lungs of asthma mouse models of different severity. J Synchrotron Rad 2015; 22: 1106-1111.

15 Paganin D, Mayo SC, Gureyev TE, et al. Simultaneous phase and amplitude extraction from a single defocused image of a homogeneous object. J Microsc 2002; 206(Pt 1): 33-40.

16 Mohammadi S, Larsson E, Alves F, et al. Quantitative evaluation of a single-distance phase-retrieval method applied on in-line phase-contrast images of a mouse lung. J Synchrotron Rad 2014; 21: 784-789. 